\title{
Monolithic 2 DOF fully compliant space pointing mechanism
}

\author{
E. G. Merriam ${ }^{1}$, J. E. Jones ${ }^{2}$, S. P. Magleby ${ }^{1}$, and L. L. Howell ${ }^{1}$ \\ ${ }^{1}$ Dept. of Mechanical Engineering, Brigham Young University, Provo, UT 84602, USA \\ ${ }^{2}$ Marshall Space Flight Center, NASA, Huntsville, AL 35812, USA
}

Correspondence to: L. L. Howell (lhowell@byu.edu)

Received: 10 April 2013 - Revised: 11 June 2013 - Accepted: 1 July 2013 - Published: 2 December 2013

\begin{abstract}
This paper describes the conception, modeling, and development of a fully compliant two-degreeof-freedom pointing mechanism for application in spacecraft thruster, antenna, or solar array systems. The design objectives and the advantages of a compliant solution are briefly discussed. Detailed design decisions to meet project objectives are described. Analytical and numerical models are developed and subsequently verified by prototype testing and measurements in several iterations. A final design of the 3-D printed titanium monolithic pointing mechanism is described in detail and its performance is measured.
\end{abstract}

\section{Introduction}

Current space mechanisms have arrived at a state of highly optimized design (Fowler et al., 2011). They are subject to many harsh environmental conditions, including thermal gradients, vibrational loading, and operation in a vacuum (Fusaro, 1999). As designers seek to reduce space mechanism size and cost, new mechanism topologies and manufacturing techniques must be considered that address these design challenges.

Compliant mechanisms have the potential to solve many of the challenges faced in space mechanism design. Additionally, advances in additive manufacturing enable the inexpensive creation of complex geometries. In this work Electron Beam Melting (EBM) was selected as the manufacturing process. Combining compliant mechanisms with EBM enables the creation of monolithic metal compliant mechanisms with complex geometry and frictionless, multi-axis motion. While many mechanism types stand to benefit from such characteristics, this work addresses the pointing mechanism.

A pointer is a mechanism that has the capability to orient an output stage along one or more axes. Pointing mechanisms have wide applications in spacecraft design. In space, the capability of pointing a thruster could eliminate the need for multiple thruster arrays, reducing part count and potential failure points. The ability to accurately point a commu- nications antenna could decrease the power required to send data. Similarly, pointing a solar array would give the ability to orient it for optimal capturing of solar radiation, increasing efficiency of energy capture. Figure 1a shows the pointer mechanism developed in an effort to demonstrate incorporating compliant mechanism design in a space-centered application.

Design objectives for the pointer are given in Table 1. It is intended that the rotational range specification be increased for future design iterations: fifteen degrees was chosen as a baseline. Repeatability must be high to eliminate the need for active position sensing. Center shift, or the distance that the axis of rotation moves as the mechanism deflects, must be low to increase pointing accuracy. Fifty hertz was chosen as a baseline target of first natural frequency; this constraint can be relaxed or increased depending on the application of the pointer. The thruster load was chosen for a standard thruster size, and the life specification is for earth-orbit satellites. Mechanism size is limited by the build envelope of the manufacturing system $(200 \mathrm{~mm}$ wide $\times 200 \mathrm{~mm}$ deep $\times 180 \mathrm{~mm}$ height).

\section{Background}

Various types of pointing mechanisms have been evaluated for use in space. The classic gimbals, or Cardan suspension, 


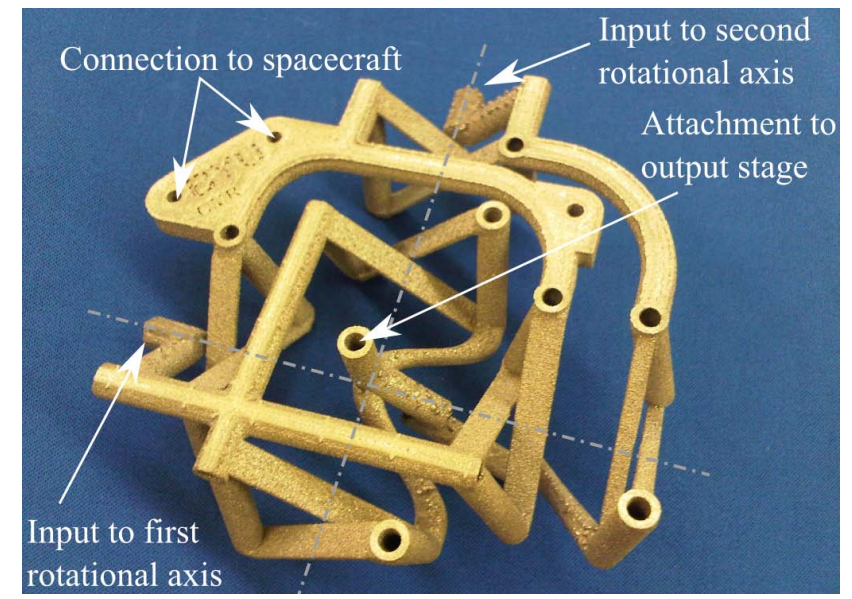

Figure 1a. Annotated photograph of a 3-D printed titanium 2 DOF pointing mechanism.

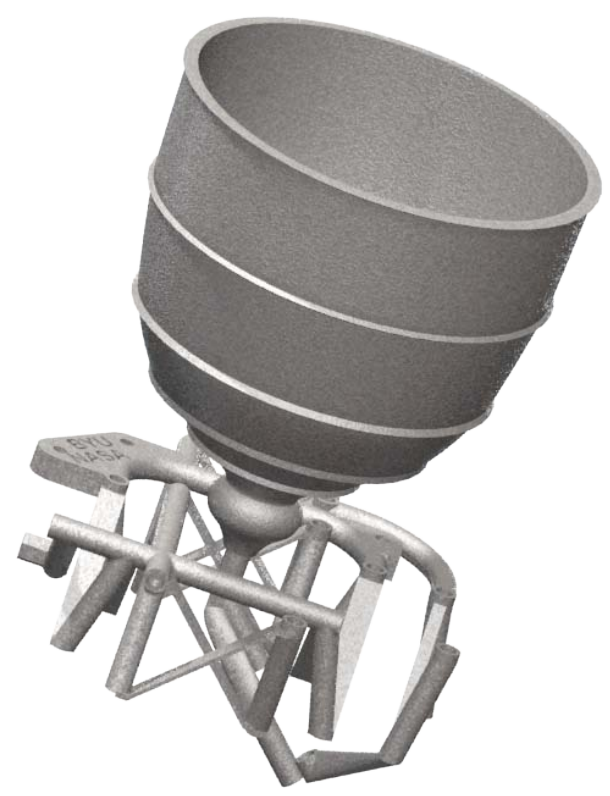

Figure $1 \mathrm{~b}$. The pointer mechanism integrated with a small attitude control thruster demonstrating compliant mechanism design in a space-centered application.

can provide good accuracy but proper arrangement of actuators is a non-trivial problem. Single-pivot pointing mechanisms using Hooke or ball-and-socket joints have several feasible configurations, but their actuation is complex. Azimuthover-elevation pointing mechanisms are essentially two tilting mechanisms mounted in series with orthogonal axes of rotation. These tend to be heavier than other pointing mechanisms and have slip rings or cables with a service loop for signal transfer (Fusaro, 1999).

Other developments have addressed the issue of positioning and orienting an object with devices such as the Agile Eye (Gosselin and Hamel, 1994; Gosselin et al., 1999) and
Table 1. Design objectives of space pointer.

\begin{tabular}{lll}
\hline Description & $\begin{array}{l}\text { Target } \\
\text { Value }\end{array}$ & $\begin{array}{l}\text { Achieved } \\
\text { Value }\end{array}$ \\
\hline Rotational range & $\pm 15^{\circ}$ & $\pm 15^{\circ}$ \\
Repeatability (\% RSD) & $<2.5 \%$ & $0.75 \%$ \\
Center Shift & $<0.5 \mathrm{~mm}$ & $0.28 \mathrm{~mm}$ \\
Thruster load & $445 \mathrm{~N}$ & $445 \mathrm{~N}$ \\
Mass & $<200 \mathrm{~g}$ & $93.2 \mathrm{~g}$ \\
First Natural Frequency & $>50 \mathrm{~Hz}$ & $55 \mathrm{~Hz}$ \\
Life (cycles) & $>1000$ & 100000 \\
Allows space for electronics/fuel lines & Yes & Yes \\
\hline
\end{tabular}

the Canfield joint (Canfield and Reinholtz, 1998). The Canfield joint has a large workspace and parallel architecture that allows wires and plumbing to be routed without risk of pinching or shearing, and served as early inspiration for this work.

This new pointer design is approached by applying the advantages of compliant mechanisms. Compliant mechanisms achieve their mobility through deflection of flexible members (Howell, 2001; Howell et al., 2013). This avoids sliding contact, eliminating friction and wear along with the need for lubrication. These improvements are especially useful when operating in a vacuum. Reducing the number of traditional joints can reduce or eliminate backlash, which is a contributing factor to the use of compliant mechanisms in precision instrumentation (Motsinger, 1964). Additionally, compliant mechanisms lend themselves to monolithic construction, giving all components an equal coefficient of thermal expansion. This avoids both binding and loss of precision due to large changes in temperature (Fusaro, 1999). Finally, compliant mechanisms can often be designed with significant weight savings when compared to traditional mechanisms (Howell, 2001).

\section{Rigid body replacement synthesis}

Numerous mechanism topologies were evaluated and a fivebar spherical mechanism was selected. It requires only two actuators and can passively support the applied thruster load. It is superior to a Cardan suspension in this application because with the addition of the driver dyad both actuators can be attached to ground, reducing rotational inertia. With proper attention to geometry, it can have the aforementioned desirable qualities of the Canfield joint, i.e. plumbing and wiring are not at risk of damage from pinching or shearing.

Rigid body replacement synthesis (Howell et al., 2013; Olsen et al., 2010) was used to convert the selected kinematic concept to a compliant mechanism. To select a compliant pivot for use in the mechanism, four pivot types were evaluated for suitability: the cross-axis flexural pivot (CAFP) (Jensen and Howell, 2002), the split-tube flexure (STF) (Goldfard and Speich, 1999), the cartwheel 


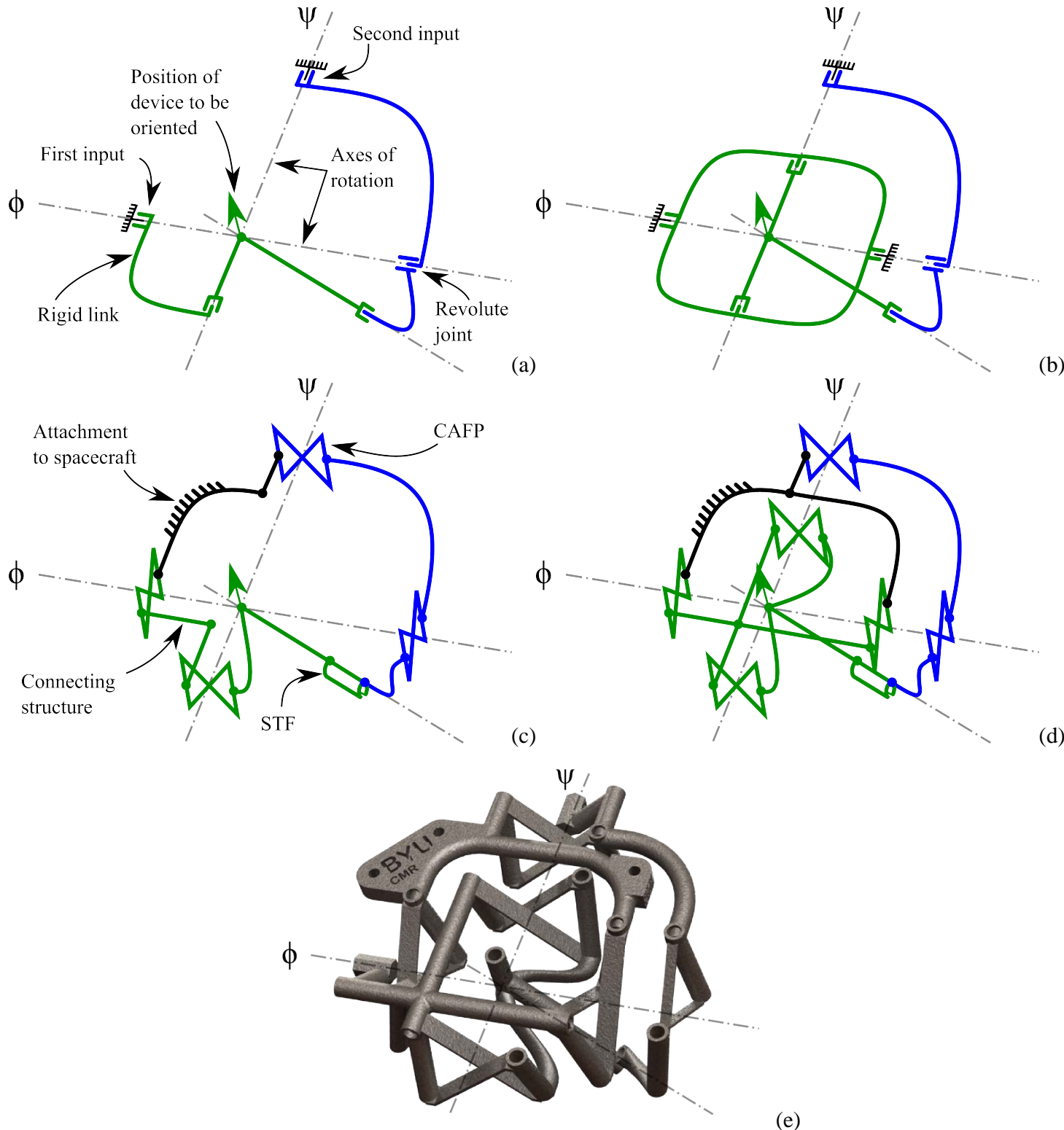

Figure 2. Rigid body replacement for the spherical five-bar mechanism. (a) Rigid link representation. The arrow is located at the intersection of the axes of the spherical mechanism and represents the mechanism to be oriented. (b) Symmetry added to increase stiffness under load. (c) Basic mechanism with CAFPs and a STF. (d) Representation of full mechanism. (e) Rendering of CAD model.

hinge $(\mathrm{CH})(\mathrm{Pei}, 2009)$, and the small-length flexural pivot (SLFP) (Howell and Midha, 1994). Metrics for comparison were stiffness, material volume, envelope, and center shift. The results are shown in Table 2. For comparison, the flexure geometry was chosen for minimum mass and equal stress $(486 \mathrm{MPa})$ at a specified deflection $\left(15^{\circ}\right)$. Joint material was assumed to be titanium. Where applicable, flexure thickness was chosen to be $1.0 \mathrm{~mm}$. Ultimately, the CAFP was selected for most rotary joints because of its low stiffness, low mass, and small envelope. The split-tube flexure was selected to replace one rotary joint because of its high off-axis stiffness and the ease with which it could be integrated into the mechanism structure. Figure 2 shows graphically the progression from rigid kinematic mechanism to the fully compliant mechanism.

In this application, flexure design is limited by space, manufacturing, stability, and stress. To increase the range of motion and decrease actuation torque, a thin, narrow, long flexure is desired. Electron Beam Melting (EBM, see Sect. 5) material data (EOS GmbH, 2008; MorrisTech, 2013; Svensson, 2009) provided an estimated ultimate tensile stress $(1034 \mathrm{MPa})$ and standard deviation (62 MPa). A conservative S-N fatigue curve (Norton, 2006) was generated for EBM produced titanium parts, shown in Fig. 3. 
Table 2. Comparison of flexures for equal stress at $15^{\circ}$ deflection.

\begin{tabular}{lrrrr}
\hline Metric & STF & CH & CAFP & SLFP \\
\hline $\begin{array}{l}\text { Torsional Stiffness } \\
\left(\mathrm{N} \mathrm{m} \mathrm{rad}^{-1}\right)\end{array}$ & 20.38 & 16.22 & 3.26 & 3.03 \\
$\begin{array}{l}\text { Material Volume } \\
\left(\mathrm{cm}^{3}\right)\end{array}$ & 3.09 & 6.27 & 0.52 & 0.52 \\
$\begin{array}{l}\text { Envelope } \\
\left(\mathrm{cm}^{3}\right)\end{array}$ & 17.79 & 294.60 & 5.24 & 0.52 \\
$\begin{array}{l}\text { Center Shift } \\
(\mathrm{mm})\end{array}$ & - & 0.20 & 0.33 & - \\
\hline
\end{tabular}

The maximum stress occurring in the CAFP can be approximated as

$\sigma=\frac{S_{\theta} \theta E t}{2 l \cos \left(45^{\circ}\right)}$

where $\sigma$ is stress, $S_{\theta}=0.965$ is a CAFP coefficient, $\theta$ is rotation, $E$ is elastic modulus, $t$ is thickness, and $l$ is segment length (Jensen and Howell, 2002). Using $\theta=0.261 \mathrm{rad}$, $E=111 \mathrm{GPa}, t=1 \mathrm{~mm}$, and $l=4.06 \mathrm{~cm}$, the stress was estimated to be $494 \mathrm{MPa}$. The $\mathrm{S}-\mathrm{N}$ curve in Fig. 3 approximates a fatigue life of 100000 cycles, which is well above the design objective of 1000 cycles. However, this estimate is for low load applications such as positioning communications or solar-power systems. When a load (such as firing a thruster) is applied to the pointer, increased stress will reduce the number of cycles to failure.

\section{Mechanism models}

Before a comprehensive model of the mechanism could be developed, it was desirable to establish accurate models of the component flexures. The flexures were modeled using commercial Finite Element Analysis (FEA) software (ANSYS). The CAFP was represented with BEAM4 elements, and this model was compared to the model presented by Jensen and Howell (2002). The STF was modeled with BEAM188 elements and defined as an arbitrary cross section. Section properties were determined from Pilkey (2002) and elementary beam theory. The STF FEA model was verified using the analytical solution by Goldfard and Speich (1999). Table 3 contains comparisons of the analytical and FEA models of both the CAFP and STF. Agreement between the FEA and analytical solutions gave confidence that the models used have a sufficient degree of accuracy.

The kinematics were derived using 3-D rotation matrices,

$\left[R_{x}\left(90^{\circ}\right)\right]\left[R_{y}(\theta)\right]\left[R_{z}\left(90^{\circ}\right)\right]\left[R_{y}(\phi)\right]=$

$\left[R_{x}\left(-90^{\circ}\right)\right]\left[R_{y}(-g)\right]\left[R_{x}(\gamma)\right]\left[R_{y}(-g)\right]\left[R_{x}(\eta)\right]\left[R_{z}\left(-90^{\circ}\right)\right]\left[R_{x}(\psi)\right]$

where variables are depicted in Fig. 4 and $g=\frac{\pi}{4}$. Multiplying the matrices gives nine equations, the following three of

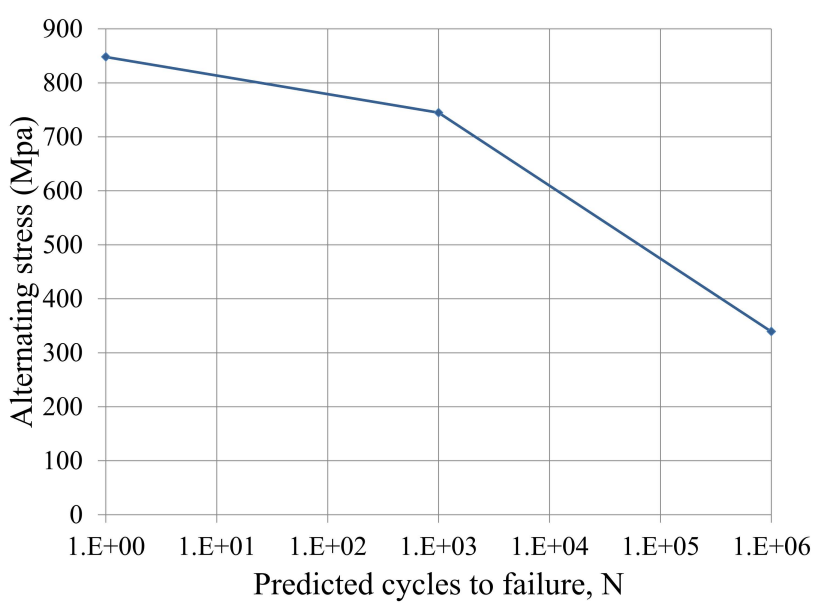

Figure 3. Estimated S-N curve for EBM produced Ti6Al4V.

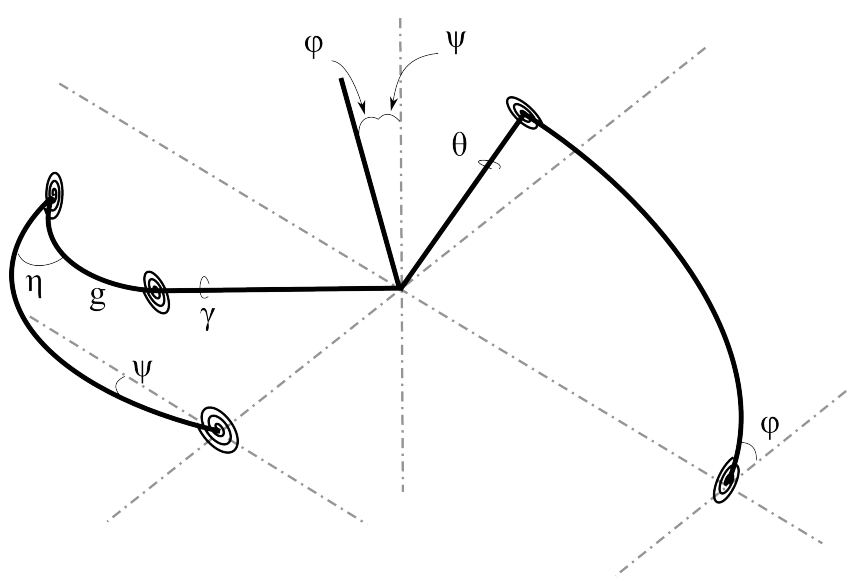

Figure 4. Angle nomenclature used to develop Eq. (2).

which can be solved for unknowns $\theta, \gamma$, and $\eta$ :

$$
\begin{aligned}
& c(\phi)=c(\gamma) c(\eta)-\frac{s(\gamma) s(\eta)}{\sqrt{2}} \\
& 0=\frac{s(\gamma) s(\psi)}{\sqrt{2}}-\left(c(\gamma) s(\eta)+\frac{s(\gamma) s(\eta)}{\sqrt{2}}\right) s(\psi) \\
& c(\theta)=\left(\frac{1}{2}+\frac{c(\gamma)}{2}\right) c(\psi)-\left(-\frac{s(\gamma) s(\eta)}{\sqrt{2}}+\left(-\frac{1}{2}+\frac{c(\gamma)}{2}\right) c(\eta)\right) s(\psi)
\end{aligned}
$$

where $\gamma$ and $\eta$ were found using function fmincon in Matlab, while $\theta$ could be found directly once $\gamma$ and $\eta$ were known.

Combining the kinematics, analytical models of the flexures, the pseudo-rigid-body model (Howell and Midha, 1994), and the principle of virtual work (Howell, 2001), an analytical model of the mechanism was developed that is 
Table 3. Comparison of FEA and analytical predictions of stiffness for CAFP and STF. All stiffnesses are in $\mathrm{N} \mathrm{m} \mathrm{rad}^{-1}$.

\begin{tabular}{lrrr}
\hline & FEA & Analytical & $\%$ Difference \\
\hline CAFP & 3.05 & 3.26 & $-6.94 \%$ \\
STF & 21.34 & 20.38 & $4.5 \%$ \\
\hline
\end{tabular}

Table 4. Modal analysis of connecting structure sections. First and second frequencies correspond to the desired degrees of freedom. Frequencies in $\mathrm{Hz}$.

\begin{tabular}{lrrr}
\hline $\begin{array}{l}\text { Natural } \\
\text { Frequency }\end{array}$ & $\begin{array}{r}\text { Solid } \\
\text { Square }\end{array}$ & $\begin{array}{r}\text { Hollow } \\
\text { Circle }\end{array}$ & $\begin{array}{r}\text { Square } \\
\text { Channel }\end{array}$ \\
\hline 1st & 15.5 & 15.6 & 14.8 \\
2nd & 19.6 & 19.7 & 19.3 \\
3rd & 55.2 & 73.8 & 50.3 \\
4th & 86.4 & 126.1 & 68.0 \\
5th & 108.5 & 135.8 & 84.2 \\
\hline
\end{tabular}

capable of predicting input torques.

$\left[\begin{array}{l}\sum_{i=0}^{n} k_{i} \tilde{\theta}_{i} \frac{\mathrm{d} \theta_{i}}{\mathrm{~d} q_{1}} \\ \sum_{i=0}^{n} k_{i} \tilde{\theta}_{i} \frac{\mathrm{d} \theta_{i}}{\mathrm{~d} q_{2}}\end{array}\right]=\left[\begin{array}{ll}\frac{\mathrm{d} \phi}{\mathrm{d} q_{1}} & \frac{\mathrm{d} \psi}{\mathrm{d} q_{1}} \\ \frac{\mathrm{d} \phi}{\mathrm{d} q_{2}} & \frac{\mathrm{d} \psi}{\mathrm{d} q_{2}}\end{array}\right]\left[\begin{array}{l}T_{\phi} \\ T_{\psi}\end{array}\right]$

The FEA flexure models were combined and expanded to represent the entire mechanism. A mesh refinement study was implemented to find an element size for a meshindependent solution. The resulting FEA model is shown in Fig. 5. Figure 6 shows FEA and analytical predictions of required torque for ten combinations of input angles. The models agree closely on $T_{\psi}$, but $T_{\phi}$ predictions show a discrepancy roughly proportional to the magnitude of the commanded angle. This discrepancy is due to parasitic motion in the FEA model; the reduced stiffness of that model results in a lower torque prediction. Damping is not modeled but may be an important issue for some conditions.

\subsection{Connecting structure}

Three section types were evaluated for use in the structure that connects the pivots: solid square, hollow round, and square channel. The section dimensions were determined by using a constant cross sectional area $\left(0.403 \mathrm{~cm}^{2}\right)$ and a wall thickness (where applicable) of $1.27 \mathrm{~mm}$. This approach allowed connecting structures of equal mass to be evaluated based on their relative stiffness only. To compare each structure's stiffness, an FEA modal analysis was conducted for each section type. The results are listed in Table 4 , and indicate that for equal mass the round tube structure is stiffer than either of the competing section types. It was found that increasing the stiffness of the connecting structure significantly improved the mechanism's precision by reducing cen-

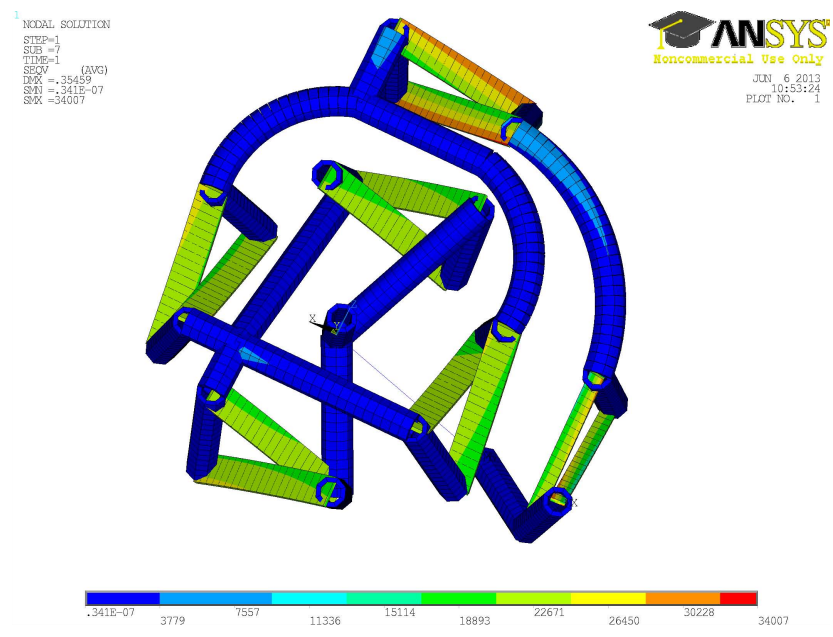

Figure 5. FEA representation of the mechanism at a deflected position. Contours are Von Mises stress in $\mathrm{lbs}^{-2}$.

ter shift (see Table 5). Although increasing structural stiffness increases stress in the flexures, the benefits of improved precision dictate that the connecting structure be as rigid as possible while still maintaining low mass. Therefore, round, hollow tubes were selected for the structure.

\section{Prototypes}

A parametric CAD model was built in the commercial package Solidworks to allow geometry to be easily altered. Mounting points were added to the ground link, and hex stubs were incorporated along the $\phi$ and $\psi$ axes to enable actuation during testing. During the testing of early prototypes, torque watches and torque wrenches were used to apply and measure actuation torques, and protractors provided angle measurement.

First prototypes were built from FlexLinks (BYU CMR Group, 2013) (custom compliant mechanism prototyping elements) and $\mathrm{LEGO}^{\circledR}$ bricks (see Fig. 7a and b). The prototype in Fig. 7b was used to further validate the FEA and analytical models, and to better visualize the mechanism behavior. The FEA model correctly predicted the torque required to deflect the prototype to several positions.

Before full-scale prototyping in metal, several titanium flexures were fabricated (see Fig. 7c and d) to examine their suitability for EBM and test their behavior. The torquedisplacement behavior of these flexures compared with FEA and analytical predictions is shown in Table 6. The differences in stiffnesses for different flexures are attributed to variations in manufactured flexure thickness. The differences between measured and predicted values is also due to high surface roughness, causing the effective thickness of the pivot to be less than the measured thickness. Applying a correction factor of 0.83 to the flexure thickness in the FEA model brings it into good agreement with measured stiffness. 


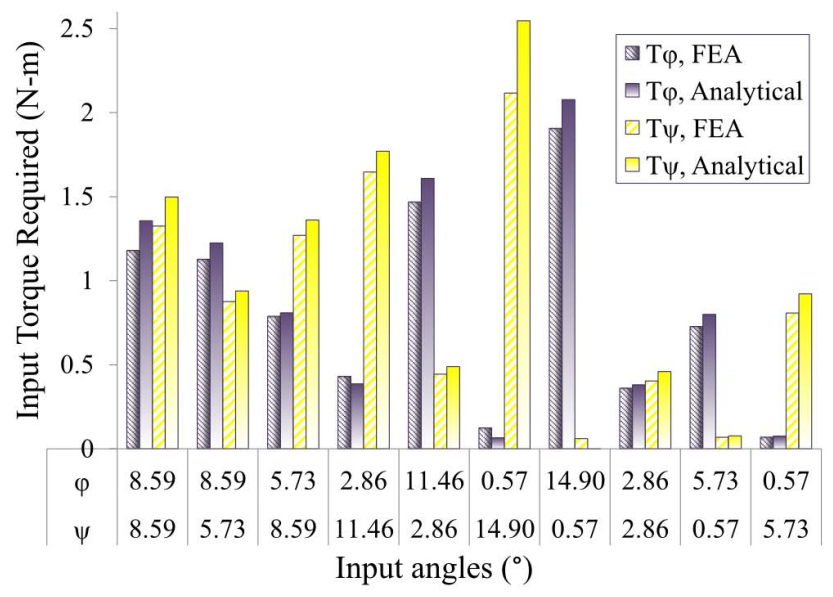

Figure 6. Comparison of FEA and analytical models at ten sets of command angles. $T_{\phi}$ and $T_{\psi}$ are torque required for given rotation about the $\phi$ and $\psi$ axes, respectively.

Table 5. Static analysis of connecting structures. All values are averages over eight representative sets of command angles. Rotational error is a root sum square difference from zero and command angles.

\begin{tabular}{llll}
\hline & $\begin{array}{l}\text { Solid } \\
\text { Square }\end{array}$ & $\begin{array}{l}\text { Hollow } \\
\text { Circle }\end{array}$ & $\begin{array}{l}\text { Square } \\
\text { Channel }\end{array}$ \\
\hline Center Shift $(\mathrm{mm})$ & 0.259 & 0.256 & 0.363 \\
Rotational Error (rad) & 0.019 & 0.017 & 0.024 \\
Max Von Mises Stress (MPa) & 453 & 475 & 422 \\
\hline
\end{tabular}

As a final check of the CAD model before creating a titanium mechanism using EBM, the mechanism was produced on an FDM printer in ABS plastic. This ABS prototype is shown in Fig. 7e. After minor cosmetic changes the design was produced in Ti6Al4V using EBM at NASA Marshall Space Flight Center. This final prototype is shown in Fig. 7f.

\section{Manufacturing considerations}

To enable the manufacture of the complex geometry in Titanium, Electron Beam Machining (EBM) was selected as the manufacturing process for the final device. EBM is a variant of 3-D printing, where the part is built from successive layers of metal powder that are melted together. This additive manufacturing process allows geometries to be created that would be difficult to fabricate using traditional methods. EBM allows parts to be designed with reduced manufacturing constraints (ArcamAB, 2013). Adherence to certain design guidelines can greatly improve part quality, and were incorporated in the space pointer to facilitate manufacturing. For example, overhanging geometry should be avoided, especially when features are thin (Cansizoglu et al., 2008). In cases where overhangs are unavoidable, support geometry should be included that is removed during post-processing.
Table 6. Predicted and measured stiffnesses of two flexure types. $\mathrm{A}, \mathrm{B}, \mathrm{C}, \alpha$, and $\beta$ designate average values of stiffness for each prototype flexure.

\begin{tabular}{ccccc}
\hline \multicolumn{5}{c}{ (a) CAFPs } \\
& FEA & A & B & C \\
Stiffness $\left(\mathrm{N} \mathrm{m} \mathrm{rad}^{-1}\right)$ & 6.0 & 5.6 & 3.8 & 4.1 \\
\hline
\end{tabular}

\begin{tabular}{llll}
\hline \multicolumn{4}{c}{ (b) STFs } \\
& FEA & $\alpha$ & $\beta$ \\
Stiffness $\left(\mathrm{N} \mathrm{m} \mathrm{rad}^{-1}\right)$ & 23.6 & 10.4 & 12.9 \\
\hline
\end{tabular}

Additionally, the wall thickness should be at least $1.0 \mathrm{~mm}$, and the maximum cross section of one build layer is $100 \mathrm{~cm}^{2}$.

\section{Testing}

To confirm that the final titanium prototype behaves as desired, a testing regime was implemented using techniques more refined than the simplified methods used for initial prototypes.

\subsection{Test method}

Custom fixtures were built to attach the mechanism to an optical breadboard. Worm-and-wheel gear sets were used to apply pure rotational displacements in series with Omega torque transducers. Transducer output was measured with a National Instruments USB carrier coupled with a LabView DAQ. Transducer calibration was accomplished by hanging masses from a lever arm of known length to apply a known, constant torque.

Stereoscopic imaging was used for position measurement because of its ability to rapidly determine locations in three dimensions. Images were captured using Dino-Lite AM3011 Digital Microscopes. The test set-up is shown in Fig. 8. Processing and position finding was done using scripts written in Matlab. Platform orientation was found by identifying the location of points A, B, and C (see Fig. 9) and using $N=$ $\boldsymbol{A} \boldsymbol{B} \times \boldsymbol{A C}$. The output angles were found as $\phi=\arctan \left(\frac{N_{x}}{N_{2}}\right)$ and $\psi=\arctan \left(\frac{-N_{y}}{N_{z}}\right)$.

Center shift was found using points $\mathrm{B}, \mathrm{C}, \mathrm{D}$, and $\mathrm{E}$ from Fig. 9. These points were chosen because they are equidistant from the center of rotation. The point locations were found for all 45 data sets. Gradient based optimization (the Matlab function fmincon) was used to determine the sphere radius, $r$, that best fit these points to its surface. Using this calculated $r$ and the undeflected positions of $\mathrm{B}, \mathrm{C}, \mathrm{D}$, and $\mathrm{E}$, a center of rotation $\left(X_{0}, Y_{0}\right.$, and $\left.Z_{0}\right)$ was also found. For each data set a relative center of rotation was found $\left(x_{0}, y_{0}\right.$, and $\left.z_{0}\right)$. The distance from this displaced center to the non-displaced center was then calculated and recorded as center shift. 

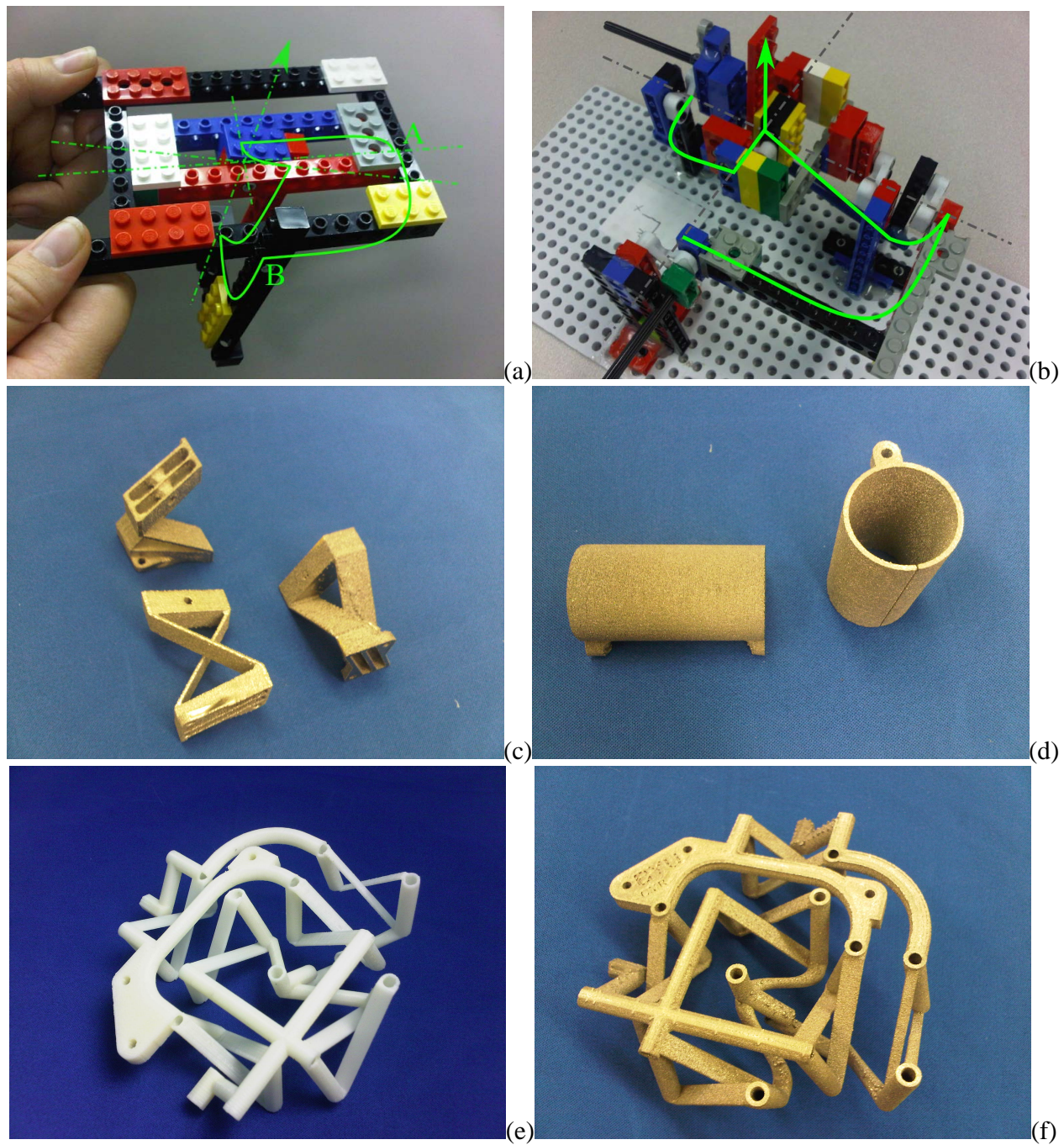

Figure 7. Titanium prototype. (a) Spherical 5-bar with central pointing platform. Rotations at A and B give two DOF. (b) Compliant prototype to confirm rigid concept. (c) Titanium CAFPs. (d) Titanium STFs. (e) Monolithic ABS prototype. (f) Titanium prototype.

Repeatability was tested by selecting four sets of command torques $T_{\phi}$ and $T_{\psi}$ and actuating the mechanism to each set four times. The rotations were measured and compared.

\subsection{Results}

To verify the FEA model, torque and displacement were measured at a variety of command angles. The flexure thickness correction factor $(0.83)$ described previously was used to correct for changes between the designed and as-fabricated flexure thicknesses. Good agreement between measured behavior and the FEA model was achieved, as shown in Figs. 10, 11, and 12. Table 1 tabulates the final values of the design metrics. All values meet or exceed the design goals. Note that rotational accuracy will depend on the method of actuation and associated control system, and was not quantified here. Center shift was used as an alternate metric of performance in lieu of rotational accuracy. This is the amount the mechanism center moves from the undeflected position. Center shift data is tabulated in Table 7, which shows that center shift is larger than anticipated but still quite small. It was found that the repeatability of the mechanism is high. Figure 13 shows that differences in measured position are less than the measurement error for four sets of command torques.

\section{Conclusions}

We have described the modeling and development of a fully compliant spherical pointing mechanism suitable for use in space applications. Monolithic construction avoids many design challenges of the space environment. Adequate precision was obtained while providing a platform capable of supporting thruster loads up to $450 \mathrm{~N}$. The design objectives in Table 1 were met or exceeded. Advantages of the mechanism 

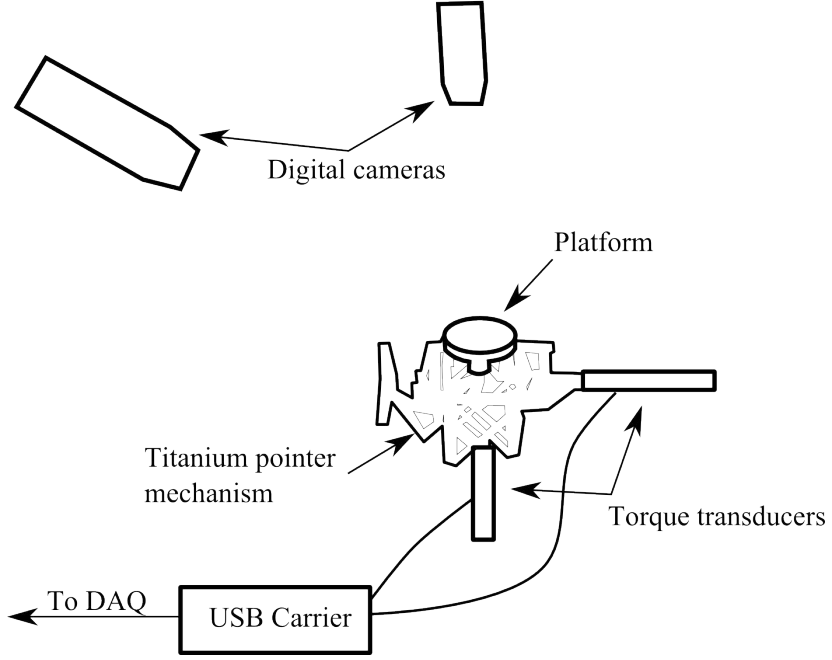

Figure 8. Test set-up for measuring applied torque and resulting displacement.

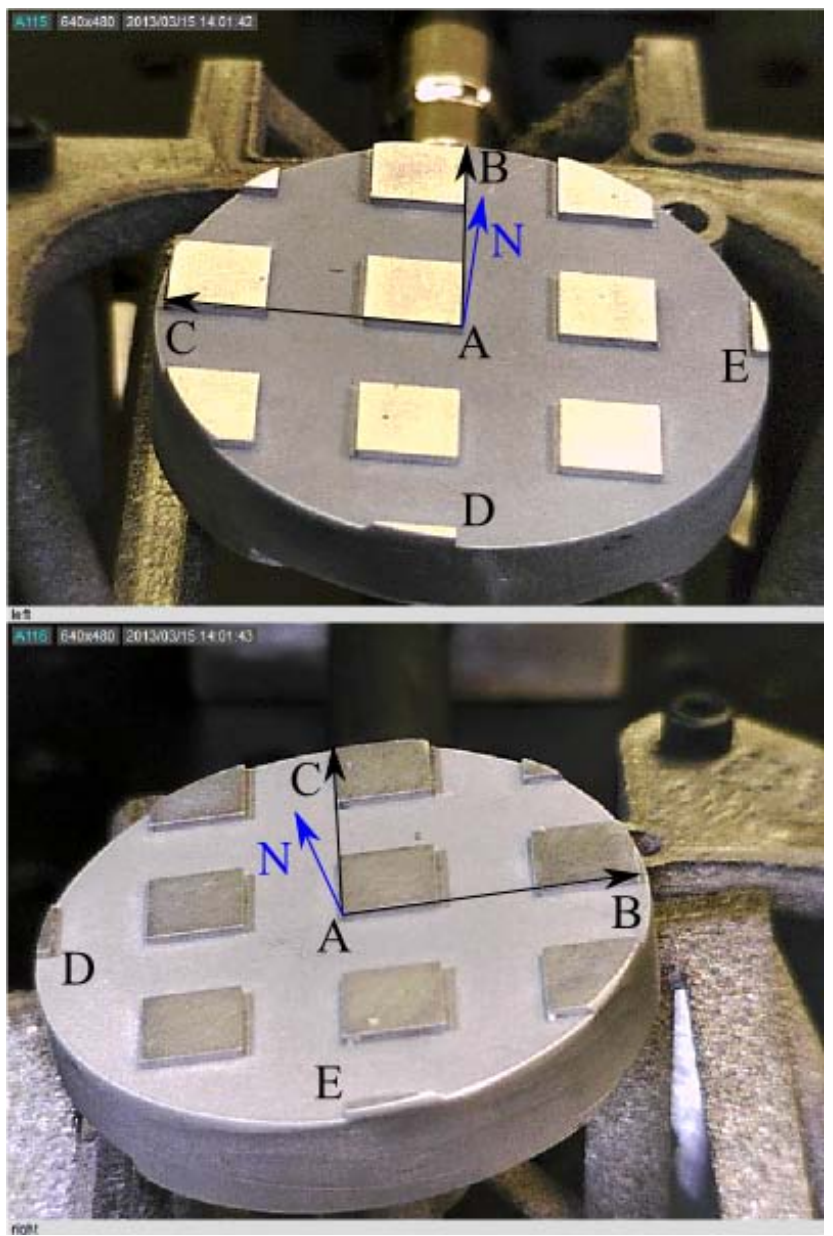

Figure 9. Points A, B, C, D, and E with vector $\boldsymbol{N}$ used for measurement of $\phi$ and $\psi$ on the titanium prototype.

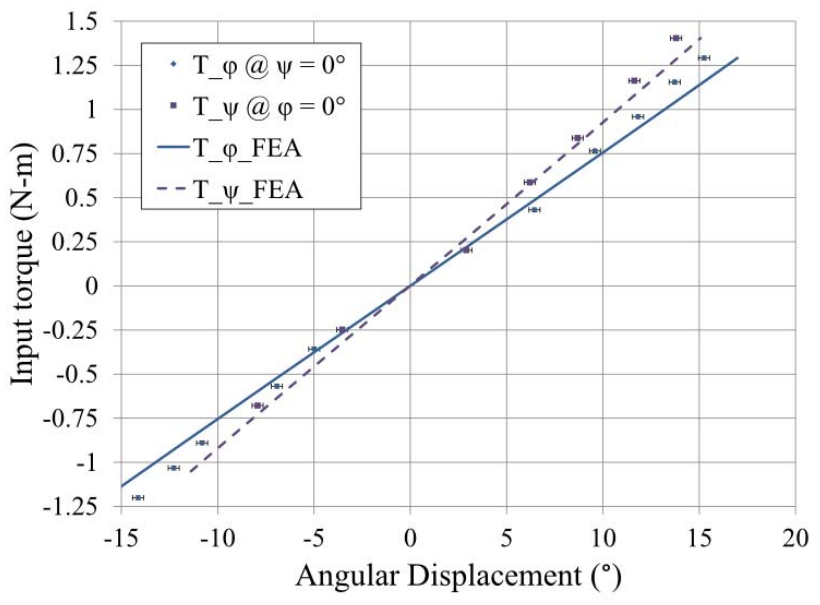

Figure 10. Comparison of measured and predicted $T_{\phi}$ and $T_{\psi}$ at $\psi=0$ and $\phi=0$, respectively for the titanium prototype.

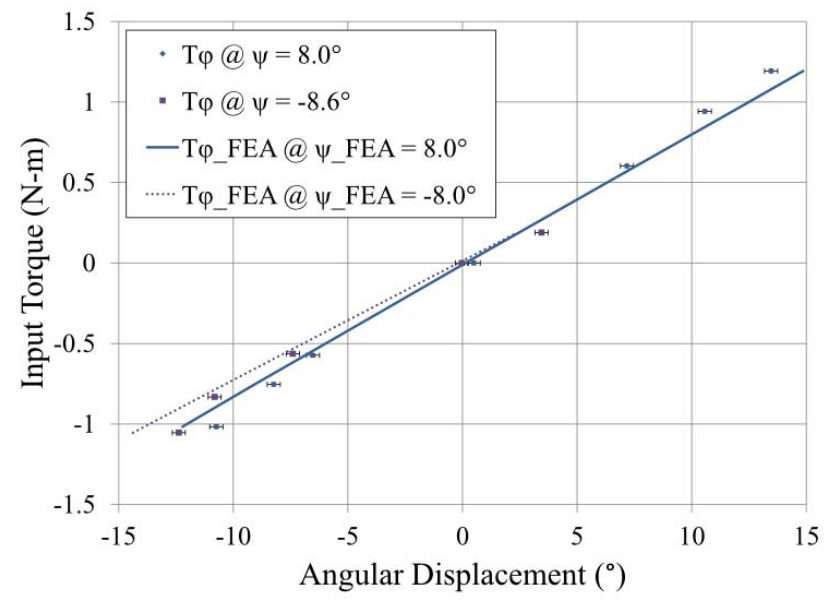

Figure 11. Comparison of measured and predicted input torques $\left(T_{\phi}\right)$ at two values of $\psi$ for the titanium prototype.

include low part count, small mass, low friction, small volume, and high reliability due to the elimination of articulating components.

The monolithic pointing mechanism could be prepared for use in a range of spacecraft applications. Figure 14 illustrates its implementation for orienting a communications antenna on a satellite. Use as a two-degree-of-freedom thruster mount was illustrated in Fig. 1b. The device enables orientation of the thruster while maintaining ready routing of required fuel lines.

The performance of the pointer mechanism shows that combining compliant mechanisms with the manufacturing capabilities of 3-D printing has the potential to influence the way space mechanisms are designed. This work shows that monolithic mechanisms can provide high performance in multi-axis systems while eliminating friction, wear, backlash, and lubrication issues. Possible future work includes 


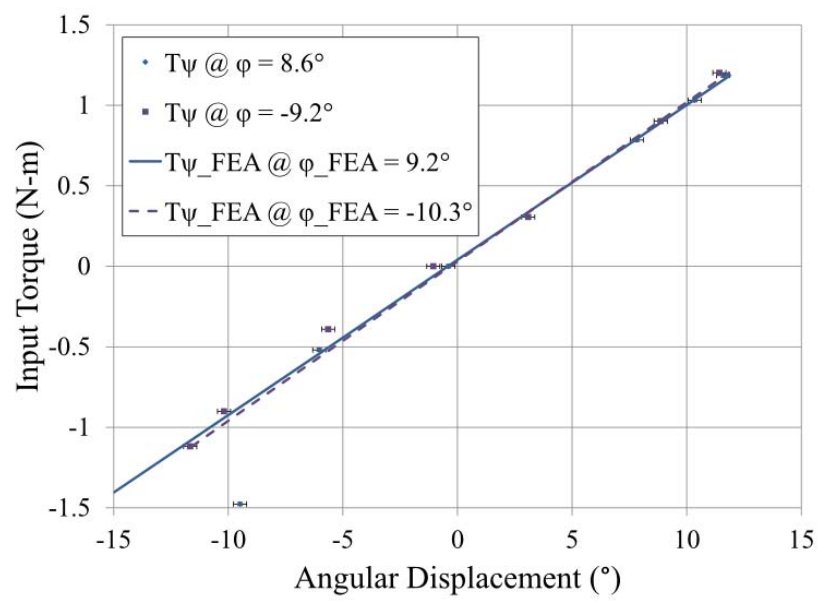

Figure 12. Comparison of measured and predicted input torques $\left(T_{\psi}\right)$ at two values of $\phi$ for the titanium prototype.

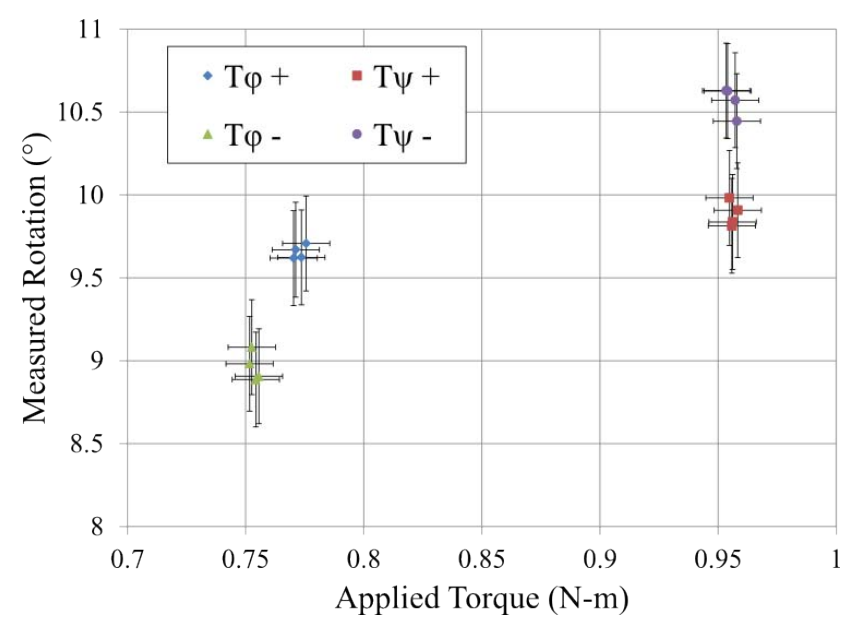

Figure 13. Measured rotation for applied torques $T_{\phi}$ and $T_{\psi}$ at $\psi=$ 0 and $\phi=0$, respectively. All data are absolute values with the sign indicated by the legend. Data is for the titanium prototype.

efforts to utilize static balancing as a strategy for reducing actuation effort, incorporating the capacity for structural damping, and optimizing for desirable frequency response. With feasibility demonstrated and the model verified, the pointer is available to be optimized for mass, volume, and other performance metrics for specific applications.

Acknowledgements. The authors acknowledge Eric Wilcox for his support in building testing apparatus, and Scott Thompson, Ph.D. for his help with the stereoscopic imaging code. This work was supported by the National Science Foundation and Air Force Office of Scientific Research through NSF Grant No. 1240417 and by NASA Marshall Space Flight Center through NASA Grant No. NNX13AF52G.

Edited by: G. Hao

Reviewed by: two anonymous referees

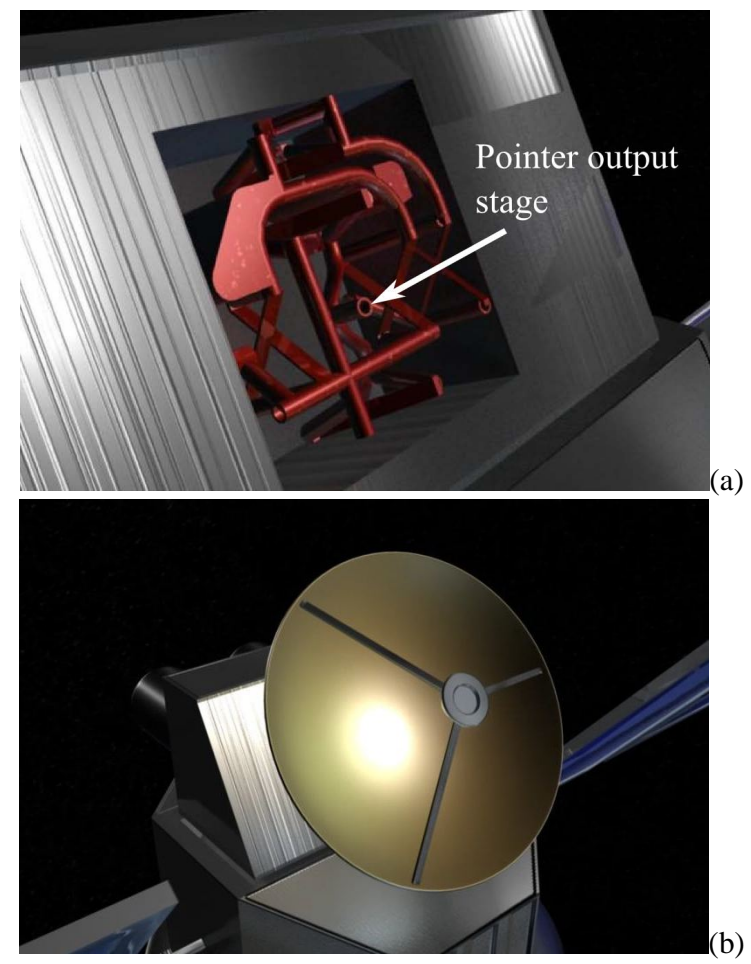

Figure 14. Possible application of the pointer for orienting a communications antenna. To see the full video, go to http://youtu.be/ xIAvIIoRw8w. (a) The pointer mechanism on a possible satellite. (b) The same satellite as in $14 \mathrm{a}$, with communications antenna mounted to the pointer output stage.

Table 7. Center shift data for analytical model, numerical model, and measured behavior. Analytical maximum center shift is a root sum squared total for a single hinge. Numerical and measured data were collected from 45 distinct sets of applied torques. All data are in millimeters.

\begin{tabular}{lccr}
\hline Metric & Analytical & Numerical & Measured \\
\hline Mean & - & 0.18 & 0.28 \\
Standard Deviation & - & 0.10 & 0.12 \\
Max & 0.57 & 0.46 & 0.63 \\
Min & - & 0.02 & 0.08 \\
\hline
\end{tabular}

\section{References}

ArcamAB: Additive Manufacturing (AM), http://www.arcam.com/ technology/additive-manufacturing/, last access: 19 November 2013.

BYU CMR Group: FlexLinks, http://compliantmechanisms.byu. edu/downloads/flexlinks, last access: 19 November 2013.

Canfield, S. and Reinholtz, C.: Development of the carpal robotic wrist, in: Experimental Robotics V, edited by: Casals, A. and DeAlmeida, A., Vol. 232 of Lecture notes in control and information sciences, 423-434, Springer-Verlag, London Ltd, 1998. 
Cansizoglu, O., Harrysson, O. L. A., West II, H. A., Cormier, D. R., and Mahale, T.: Applications of structural optimization in direct metal fabrication, Rapid Prototyping J., 14, 114-122, 2008.

Fowler, R. M., Howell, L. L., and Magleby, S. P.: Compliant space mechanisms: a new frontier for compliant mechanisms, Mech. Sci., 2, 205-215, doi:10.5194/ms-2-205-2011, 2011.

Fusaro, R.: NASA space mechanisms handbook and reference 342 guide, NASA/TP-1999-206988, 1999.

Goldfarb, M. and Speich, J. E.: A well-behaved revolute flexure joint for compliant mechanism design, J. Mech. Des., 121, 424 429, 1999.

Gosselin, C. and Hamel, J.: The agile eye: A high-performance three-degree-of-freedom camera-orienting device, in: 1994 IEEE International Conference on Robotics and Automation, 781-786, IEEE, 1994.

Gosselin, C. and Caron, F.: Two degree-of-freedom spherical orienting device, US Patent 5,966,991, 1999.

Hopkins, J. and Culpepper, M.: Synthesis of multi-degree of freedom, parallel flexure system concepts via Freedom and Constraint Topology (FACT) - Part I: Principles, Precis. Eng., 34, 259-270, 2010.

Howell, L.: Compliant Mechanisms, John Wiley \& Sons, Inc., 2001.

Howell, L. and Midha, A.: A method for the design of compliant mechanisms with small-length flexural pivots, J. Mech. Des., 116, 280-290, 1994.

Howell, L. L., Magleby, S. P., and Olsen, B. M.: Handbook of Compliant Mechanisms, Wiley, 2013.

Jensen, B. and Howell, L.: The modeling of cross-axis flexural pivots, Mech. Mach. Theory, 37, 461-476, 2002.
Morris Technologies, I.: Titanium Ti64, www.morristech.com, last access: 19 November 2013.

Motsinger, R.: Flexural devices in measurement systems, Measurement Engineering, 1, 383-435, 1964.

Norton, R. L.: Machine Design An Integrated Approach, 3E, Pearson Prentice Hall, 2006.

Olsen, B. M., Issac, Y., Howell, L. L., and Magleby, S. P.: Utilizing a Classification Scheme to Facilitate Rigid-Body Replacement for Compliant Mechanism Design, Proceedings of the ASME International Design Engineering Technical Conferences, Montreal, Quebec, 15-18 August 2010, Paper No. DETC2010-28473, 2010.

Pei, X., Yu, J., Zong, G., Bi, S., and Su, H.: The modeling of cartwheel flexural hinges, Mech. Mach. Theory, 44, 1900-1909, 2009.

Pilkey, W. D.: Analysis and design of elastic beams, John Wiley \& Sons, Inc., 2002.

EOS GmbH - Electro Optical Systems: EOS titanium Ti64 for EOSINT M 270 systems (titanium version), www.eos.info, D82152 Krailling/München, 2008.

Svensson, M.: Material properties of EBM-manufactured Ti6Al4V \& Ti6Al4V ELI under RAW and HIP conditions, Arcam AB, 2009.

Vakili, V. and Shu, L.: Towards biomimetic concept generation, in: Proceedings of the ASME Design Engineering Technical Conference, 4, 327-335, 2001. 